\title{
The dust disk of HR 4049
}

\section{Another brick in the wall}

\author{
C. Dominik ${ }^{1}$, C. P. Dullemond ${ }^{2}$, J. Cami ${ }^{1,3}$, and H. van Winckel ${ }^{4}$ \\ 1 Sterrenkundig Instituut “Anton Pannekoek", Kruislaan 403, 1098 SJ Amsterdam, The Netherlands \\ e-mail: dominik@science.uva.nl \\ 2 Max Planck Institut für Astrophysik, Karl Schwarzschild Strasse 1, 85748 Garching, Germany \\ e-mail: dullemon@mpa-garching.mpg.de \\ 3 SRON-Groningen, PO Box 800, 9700 AV Groningen, The Netherlands \\ e-mail: cami@astro.rug.nl \\ ${ }^{4}$ Instituut voor Sterrenkunde, Katholieke Universiteit Leuven, Celestijnenlaan 200 B, 3001 Heverlee, Belgium \\ e-mail: Hans.VanWinckel@ster. kuleuven.ac. be
}

Received 6 March 2002 / Accepted 8 October 2002

\begin{abstract}
We present the Spectral Energy Distribution of HR 4049 based on literature data and new continuum measurements at $850 \mu \mathrm{m}$. The SED shows variable absorption in the UV, and a large IR excess, both caused by circumstellar dust. The shape of the IR excess from $1 \mu \mathrm{m}$ all the way down to $850 \mu \mathrm{m}$ can be nearly perfectly fitted with a single blackbody function at $T \approx 1150 \mathrm{~K}$ or alternatively with a sum of blackbodies in a narrow temperature range. The energy emitted in this IR continuum radiation is about one-third of the stellar luminosity. We show that this blackbody radiation must be due to the presence of a circumbinary disk with a large height. This disk must also be gas-rich, in agreement with the observations of molecular bands in the ISO-SWS spectrum. We present two possible scenario's for explaining the shape and the intensity of the IR excess. The first scenario involves large grains $(a \geq 1 \mathrm{~mm})$ that each radiate like a blackbody. The second scenario argues that the blackbody radiation is due to a very optically thick circumbinary disk. We investigate if such a disk would indeed produce blackbody radiation by presenting results from radiative transfer calculations. We further quantify the properties of such a disk and its stability in the framework of (hydro)dynamics, grain settling, radiation pressure and grain drift. The virtues and shortcomings of both models for the origin of the IR blackbody are discussed by contrasting them with other observations and assessing them in the framework of (binary) (post-)AGB evolution.
\end{abstract}

Key words. circumstellar matter - infrared: stars - binaries: spectroscopic - stars: evolution - stars: variables: general

\section{Introduction}

When low- to intermediate mass stars leave the Asymptotic Giant Branch (AGB), they spend a relatively short time $\left(10^{3}\right.$ to $10^{4}$ years) on their way to become a planetary nebula; this stage of stellar evolution is the post-AGB phase. During this phase, the stellar interior is gradually more exposed, so that the effective temperatures increase from typical AGB values $(\sim 3000 \mathrm{~K})$ to those commonly found in planetary nebulae $(\sim 30000 \mathrm{~K})$. At the same time, the luminosity of these stars remains more or less constant and is generally in the range $10^{3}-5 \times 10^{4} L_{\odot}$. As the AGB phase is characterized by substantial mass-loss, it is expected that post-AGB stars are surrounded by circumstellar matter. It is this material that might later on become visible as a planetary nebula.

Send offprint requests to: $\mathrm{C}$. Dominik, e-mail: dominik@science.uva.nl
HR 4049 is considered to be the prototype of a group of post-AGB stars sharing similar - but rather peculiar characteristics (see van Winckel et al. 1995). They are all members of a (close) binary system, often with highly eccentric orbits. Their photospheres show a high degree of metal-depletion, while volatile elements as $\mathrm{CNO}, \mathrm{S}$ and $\mathrm{Zn}$ show solar-like abundances. Most of them exhibit a deficiency in the UV and optical, while they do have a - sometimes huge - IR excess, pointing to the presence of circumstellar dust which thermally re-emits the radiation it absorbs in the UV. The key to explaining all of these peculiarities is probably the binary nature of these objects which allows the formation of a massive, stable and long-lived circumbinary disk. The formation of dust in these disks followed by re-accretion of the depleted gas onto the stellar photosphere explains the photospheric abundance patterns (Waters et al. 1992). Moreover, the interplay of a massive circumbinary disk with the central binary might be important for the dynamics of the binary system (Mellema 1997; 
Icke 1988). The lead role of the dust disks in these systems can therefore hardly be overestimated.

HR 4049 was first suggested to be an object in the transition phase from the AGB to the Planetary Nebula stage by Lamers et al. (1986) who discovered both a large IR excess and severe UV deficiency indicating the presence of circumstellar dust. From a variety of observational results, it is clear that the properties of this circumstellar dust are unusual.

Waelkens et al. (1991) showed that photometric variations are correlated with radial velocity variations: the star is faintest and reddest when the star is at inferior conjunction. The most plausible explanation for this phenomenon is that the dust which is responsible for the extinction is not distributed in a spherical shell, but concentrated in a disk surrounding the entire binary system - a circumbinary disk - observed at a rather high inclination: in such a geometry the amount of dust in the line of sight is largest at inferior conjunction which explains both the photometry and color variations.

The amplitude of the circumstellar extinction is wavelength-dependent, and scales linearly with $\lambda^{-1}$ (Waelkens et al. 1991; Waters et al. 1989; Buss et al. 1989). The extinction remains linear in the UV and shows no sign of the $2175 \AA$ bump (Buss et al. 1989). A linear spectral extinction can be understood in terms of Mie scattering when the size of the dust grains is small compared to the wavelength. This yields an upper limit of $300 \AA$ for the size of the dust grains in the line-of-sight towards HR 4049. Joshi et al. (1987) and Johnson et al. (1999) presented spectropolarimetry in the UV and the optical. The data show a high degree of polarization in the UV, with a change of $90^{\circ}$ in the position angle around $2000 \AA$ - the signature of scattering material in a bipolar distribution. The polarization in the optical seems to vary with orbital phase (Johnson et al. 1999), suggesting that the polarization in the optical is due to scattering in the circumbinary disk. In the $\mathrm{UV}$, the polarization is then caused by scattering in the bipolar lobes, which should contain a population of small grains $(a<0.05 \mu \mathrm{m})$.

Waters et al. (1989) studied the properties of the circumstellar dust around HR 4049. They detected emission features attributed to Polycyclic Aromatic Hydrocarbons (PAHs); the ISO/SWS spectrum of this object (Beintema et al. 1996) shows these features in great detail, suggestive of a C-rich dust composition. This is further corroborated by Geballe et al. (1989) who studied the profiles of the 3.43 and $3.53 \mu \mathrm{m}$ emission features recently identified with vibrational modes of hydrogenterminated crystalline facets of diamond (Guillois et al. 1999).

In an accompanying paper (Paper I hereafter, in preparation) we analyze the molecular bands found in the ISO/SWS spectrum. Although the dust features present in the spectrum can all be attributed to C-rich components, the molecular species are typical for the composition found around an O-rich AGB star. The dust surrounding HR 4049 therefore seems to be $\mathrm{C}$-rich, while the gas is more O-rich in nature.

Another intriguing property of the ISO/SWS spectrum is that the entire IR continuum can be matched nearly perfectly with a blackbody at a single temperature of $1200 \mathrm{~K}$. An extrapolation of this blackbody also matches the $60 \mu \mathrm{m}$ IRAS point.
In this paper we (re-)investigate the circumstellar environment of HR 4049. In Sect. 2 we present the Spectral Energy Distribution (SED) of HR 4049 from the UV to submm wavelengths, showing that the dust continuum remains a blackbody to the longest wavelengths observed so far. In Sect. 3 we show that the SED can be used to derive strong constraints on the circumstellar dust distribution. We conclude that there are only two possible dust distributions - both having a disk geometry - that can meet these observational constraints; these are described in Sects. 4 and 5. The properties of the circumstellar dust disk within the framework of these two possible scenarios are addressed in Sect. 6. In Sect. 7 we compare the two possibilities in the framework of disk dynamics and stellar evolution, and assess the plausibility of both models in the framework of other observations presented in the literature.

\section{The Spectral Energy Distribution of HR 4049}

To compose the Spectral Energy Distribution (SED) of HR 4049, we used UV, optical and near-IR observations found in the literature, data obtained with the Short Wavelength Spectrometer (SWS, de Graauw et al. 1996) on board the Infrared Space Observatory (ISO, Kessler et al. 1996) and new data obtained with the James Clerk Maxwell Telescope (JCMT).

The UV part of the SED is based on the many measurements of HR 4049 by the International Ultraviolet Explorer (IUE) (see e.g. Monier \& Parthasarathy 1999). Ground-based optical and near-IR photometry observations were acquired over a long period; the observations we use in this paper are basically the same as presented in e.g. van Winckel (2001). In the IR, we use the ISO/SWS spectrum from 2.3 to $30 \mu \mathrm{m}$ (see Paper I) and IRAS photometry points at 12, 25, 60 and $100 \mu \mathrm{m}$. Note however that the $100 \mu \mathrm{m}$ point is an upper limit; this observation is probably contaminated by interstellar cirrus clouds. The $60 \mu \mathrm{m}$ point is not contaminated because the emission at this wavelength is clearly a point source (Waters et al. 1989).

In this paper we report upon very recent observations carried out with the JCMT using the Submillimetre Common-User Bolometer Array (SCUBA) to obtain a continuum measurement at $850 \mu \mathrm{m}$. HR 4049 was observed 3 times (31 Jan. 2001, 9 Oct. 2001 and 23 Nov. 2001). The data were reduced using Uranus as a calibrator; the atmospheric opacity at $850 \mu \mathrm{m}$ is fitted with a straight line at epochs between the observations of photometric calibrators. The total integration time was $2700 \mathrm{~s}$ and yields a flux of $10.4 \pm 2.2 \mathrm{mJy}$. We included this point in the SED.

As HR 4049 shows considerable photometric variations from the UV to the near-IR (e.g. Waelkens et al. 1991), we composed a SED for both photometric maximum and for photometric minimum.

The best estimate for the distance to HR 4049 is given by the Hipparcos parallax of $0.150 \pm 0.064$ mas which corresponds to a distance range between 467 and $1162 \mathrm{pc}$. To study the properties of the circumstellar dust, we first need to correct the SED for interstellar extinction. This correction is somewhat uncertain, as the extinction towards HR 4049 contains both a (variable) circumstellar and an interstellar component. 


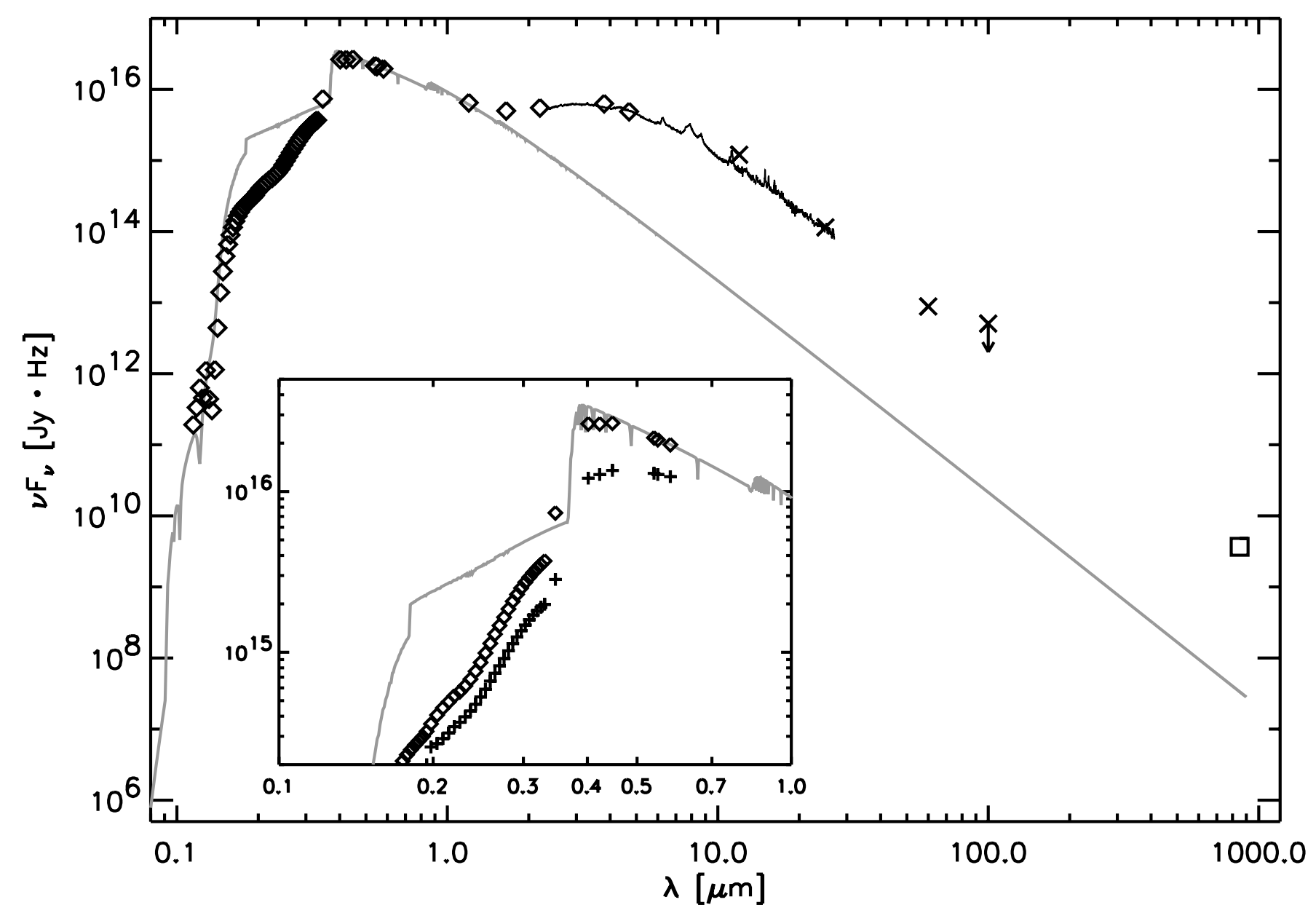

Fig. 1. The SED of HR 4049 at photometric maximum. The diamonds show IUE measurements, optical and near-IR photometry; these measurements are de-reddened using $E(B-V)=0.1$. IRAS observations at $12,25,60$ and $100 \mu \mathrm{m}$ are shown as crosses. Note that the $100 \mu \mathrm{m}$ point is only an upper limit. The continuum measurement at $850 \mu \mathrm{m}$ based on the new SCUBA observations is represented as the square. The ISO-SWS spectrum between 2.3 and $27 \mu \mathrm{m}$ is plotted as the solid black line. The thick grey line shows a Kurucz model for the star with parameters $T_{\text {eff }}=7500 \mathrm{~K}, \log g=1$ and $[\mathrm{Fe} / \mathrm{H}]=-4.5$. This Kurucz model is scaled to match the SED at photometric maximum at $580 \mathrm{~nm}$. The inset shows the SED at photometric maximum (diamonds) and photometric minimum (+) at UV and optical wavelengths, revealing (variable) extinction from circumstellar dust.

As the color variations are in phase with the photometric and radial velocity variations, the circumstellar reddening is lowest at photometric maximum. To minimize the effects of circumstellar extinction, we therefore used the SED at photometric maximum to estimate the interstellar reddening.

We estimated the interstellar component of the $E(B-V)$ by using the average interstellar extinction law given by Savage \& Mathis (1979) to de-redden the observed SED at photometric maximum and subsequently comparing this de-reddened SED with a Kurucz model. Several authors derived stellar parameters for HR 4049 using different methods (see Bakker et al. 1998, for an overview); we used these parameters and compared the de-reddened SED therefore with a Kurucz model with $T_{\text {eff }}=7500 \mathrm{~K}, \log g=1$ and $[\mathrm{Fe} / \mathrm{H}]=-4.5$. We found that adopting an interstellar reddening of $E_{\mathrm{IS}}=0.1$ yields the best fit in the optical between the SED at photometric maximum and the Kurucz model. This value for the interstellar reddening toward HR 4049 agrees well with the reddening determined for nearby stars which was found to be $0.09<E_{\mathrm{IS}}(B-V)<0.17$ (Buss et al. 1989). We adopted the same value for the $E_{\mathrm{IS}}(B-V)$ at photometric minimum. Figure 1 shows the resulting dereddened SEDs at photometric maximum and minimum and the Kurucz model. Note that the plot shows a clear UV of the dereddened spectrum compared to the Kurucz model with appropriate metallicity, a clear sign of anomalous circumstellar extinction which is also variable with orbital phase (Waelkens et al. 1995; Monier \& Parthasarathy 1999). In Fig. 2 we have subtracted the stellar model from the SED in order to show only the IR excess.

\section{Constraints on the dust distribution}

\subsection{Energy considerations}

The SED shows a UV deficiency and an IR excess, both caused by circumstellar dust. The circumstellar dust absorbs the stellar radiation in the UV producing the UV deficiency. The IR excess is caused by grains which are heated by stellar radiation and re-emit this energy thermally. It is important to realize (and often overlooked) that the IR excess is produced by all emitting 
dust grains in the beam, while the UV deficit is only due to absorption by dust grains in the line-of-sight. The source of the UV deficit and the IR excess are therefore not necessarily the same dust particles.

To get a feeling for the amount of absorption in the UV and the IR emission of the dust, we calculate the amount of energy absorbed in the UV and emitted in the IR by numerically integrating (parts of) the SEDs and the Kurucz model and express these energies in units of the stellar flux. All integrations were done using a five-point Newton-Cotes integration formula.

We first calculated the stellar flux $F_{\star}$ by integrating the Kurucz model between $145 \mathrm{~nm}$ and $850 \mu \mathrm{m}$. The energy radiated at shorter and longer wavelengths is only a negligible fraction $\left(\lesssim 10^{-4}\right)$ of the total stellar flux. To calculate the amount of energy absorbed, we should integrate the SEDs without the IR excess at both photometric maximum and minimum in order to find the fluxes $F^{\max }$ and $F^{\min }$, respectively. The IR excess dominates at wavelengths longer than $1.2 \mu \mathrm{m}$; the stellar flux at these wavelengths however still contributes about $10 \%$ of the total stellar luminosity. Fortunately the extinction becomes small at those wavelengths. We therefore assumed that the intrinsic SED at these wavelengths is equal to the Kurucz model. We then find

$1-\frac{F^{\max }}{F_{\star}}=0.04$

$1-\frac{F^{\text {min }}}{F_{\star}}=0.38$

where the superscripts refer to photometric maximum and minimum respectively. At photometric maximum, the dust in the line-of-sight thus absorbs $4 \%$ of the stellar radiation while at photometric minimum this amounts to $38 \%$. We can also use the SED to get an estimate of the optical depth:

$$
\tau=-\ln \frac{I_{\mathrm{obs}}}{I_{\text {Kurucz }}} \text {. }
$$

If the optical depth were grey, we can use Eqs. (1) and (2) to find that $\tau_{\max } \approx 0.04$ and $\tau_{\min } \approx 0.48$. However, the extinction in the UV is not grey (Buss et al. 1989) and therefore the optical depth in the UV will be larger than these grey values. We used Eq. (3) to get an idea about the actual (wavelength-dependent) optical depth and found that the optical depth reaches maximum values of $\tau \sim 1.5-2$ between 180 and $200 \mathrm{~nm}$ at photometric maximum, and even larger values $(\tau \sim 2-2.5)$ at photometric minimum.

Integrating over the IR excess yields

$\frac{F_{\mathrm{IR}}}{F_{\star}} \approx 0.32 \approx \frac{1}{3}$

so the energy radiated by the dust in the IR is about one third of the energy radiated by the star at all wavelengths! It therefore seems that the UV deficiency at minimum light and the IR excess both amount to about one third of the stellar flux. Looking only at the observations at minimum light, one might therefore be tempted to conclude that the dust is distributed spherically around the star and that the line-of-sight we probe with the UV absorption is representative. However, the large variation of the

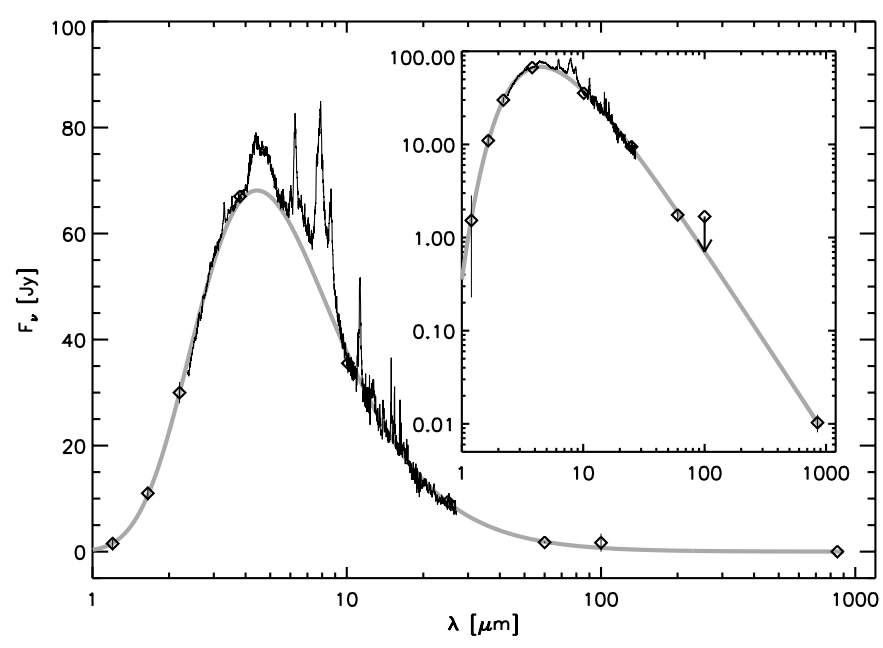

Fig. 2. The IR-excess of HR 4049. The Kurucz model is subtracted from the SED shown in Fig. 1. Errors on the near-IR photometry points, the IRAS measurements and the SCUBA point are indicated, but are generally smaller than the symbol size. Note that the "photometric" points at $3.8 \mu \mathrm{m}$ and $10 \mu \mathrm{m}$ were derived from the ISO spectrum (see text for more details). The prominent features in the ISOSWS spectrum are mainly due to PAHs, nano-diamonds, and $\mathrm{CO}_{2}$ (see Paper I). The thick grey line shows a single blackbody at a temperature of $1150 \mathrm{~K}$. The inset shows that this single temperature blackbody provides a perfect fit to the entire continuum, including the $60 \mu \mathrm{m}$ and $850 \mu \mathrm{m}$ measurements.

UV extinction with phase in fact tells us that the similar numbers are purely coincidental. We will get back to this in the discussion.

\subsection{Dust temperatures}

In Fig. 2 we have subtracted the stellar model from the SED in order to show only the IR dust emission. It shows a shape remarkably close to a blackbody over almost 3 orders of magnitude in wavelength, from 1.2 to $850 \mu \mathrm{m}$. As we showed in Paper I, a Planck function at a single temperature of $T \simeq$ 1200 provides an excellent fit to the IR continuum up to $60 \mu \mathrm{m}$. In this section, we will further quantify and constrain this dust temperature.

The first question to answer is if a single temperature blackbody can really provide a good fit to the continuum in the statistical sense. We therefore decided to perform a $\chi^{2}$ minimization. As the ISO-SWS spectrum shows a wealth of features due PAHs, nano-diamonds and molecular bands (see Paper I), we preferred not to use the spectrum for this purposes and only restrict ourselves to the near-IR photometry observations, the IRAS measurements and the SCUBA point. However, when comparing the $L$ and $M$ band observations (3.8 and $4.7 \mu \mathrm{m}$ ) with the ISO spectrum, it is seen that the fluxes of the photometric points are slightly higher than the ISO-SWS continuum; the photometric points are likely influenced by the strong emission from the $\mathrm{CO}_{2}$ stretching band which is centered around $4.2 \mu \mathrm{m}$. Also the $12 \mu \mathrm{m}$ IRAS point is not representative for the continuum (see Fig. 1); in this case, the culprit is probably the strong 11.6 PAH feature. Given these considerations, we decided not to use these photometric points. Instead, we used the ISO-SWS spectrum to obtain "photometric" continuum points 


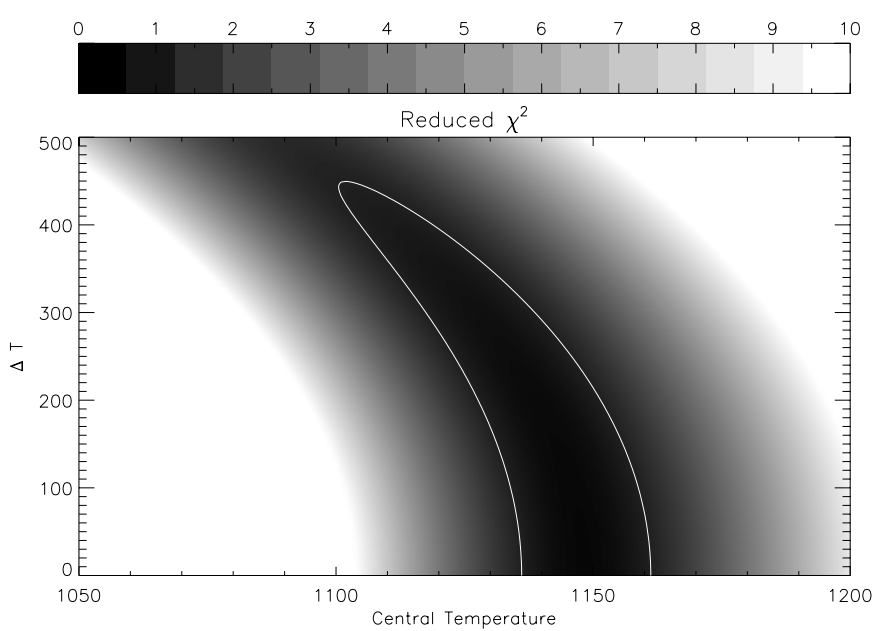

Fig. 3. $\chi_{v}^{2}$ contours for a sum of equally weighted blackbody functions. The horizontal axis is the central temperature in this sum, the vertical axis is the temperature range around this central temperature; the $\Delta T=0$ case are single temperature blackbodies. The white contour is the $\chi_{v}^{2}=1$ level, indicating that such a sum of blackbodies is indistinguishable from a single temperature blackbody even if the temperature range is as broad as $400 \mathrm{~K}$.

around these wavelengths. We replaced the $L$ band photometric point with the mean flux in the ISO-SWS spectrum between 3.7 and $3.9 \mu \mathrm{m}$; this range seems to be free of spectral features. The $12 \mu \mathrm{m}$ IRAS measurement was replaced by the mean flux between 10 and $10.2 \mu \mathrm{m}$. It is somewhat uncertain whether this is really a good continuum point, as the PAH features might still contribute to the flux at these wavelengths. It is however a much better continuum point than the IRAS $12 \mu$ m one.

We next scaled a single temperature blackbody to the $K$-band flux $(2.2 \mu \mathrm{m})$ and calculated $\chi_{v}^{2}$ (reduced $\left.\chi^{2}\right)$. We found that a single temperature blackbody can indeed accurately reproduce the continuum $\left(\chi_{v}^{2}<1\right)$ provided the temperature of this blackbody is between 1135 and $1160 \mathrm{~K}$ with a nominal best fit around $1150 \mathrm{~K}\left(\chi_{v}^{2} \approx 0.3\right)$. Figure 2 shows that this single temperature blackbody does indeed convincingly reproduce the entire IR-submm continuum.

If the IR emission is really produced by dust grains, it is unlikely that all the dust is really emitting at a single temperature. Rather, a distribution of dust centered at a central temperature $T_{\mathrm{c}}$ with a small temperature range $\Delta T$ around it would likely produce a continuum that mimics a single temperature blackbody. In order to get a feeling for the allowable range of temperatures, we therefore performed a second set of $\chi^{2}$ minimizations where we fitted the observations with a sum of equally weighted blackbodies with temperatures between $T_{\mathrm{c}}-\frac{\Delta T}{2}$ and $T_{\mathrm{c}}+\frac{\Delta T}{2}$. The $\chi_{v}^{2}$ contour map is shown in Fig. 3. As can be seen from this Figure, the allowable temperature range for such a sum of blackbodies is

$T_{\min } \geq 880 \mathrm{~K}$

and

$T_{\max } \leq 1325 \mathrm{~K}$

corresponding to a temperature range of about $450 \mathrm{~K}$, centered around $1100 \mathrm{~K}$. Dust grains with a grey opacity and with a radial power-law number density distribution $N(R) \propto R^{-\alpha}$ would imply that we have to give weights to the blackbody functions at different temperatures, with $W(T) \propto T^{2 \alpha}$. We also made a $\chi^{2}$ fit for a $\alpha=2$ and find temperature limits of 730 and $1285 \mathrm{~K}$.

\subsection{Geometrical constraints}

The main goal of this paper is to investigate which kind of dust distribution could reproduce the blackbody shape and the luminosity of the IR excess. Waters et al. (1989) actually modeled the IR excess with a spherical dust shell composed of small dust grains. This model could reproduce the IR excess down to $100 \mu \mathrm{m}$, showing that a fine-tuned distribution of small dust grains can result into a blackbody like SED. However, in order to achieve the blackbody slope at long wavelengths, the dust distribution must be very extended since the contribution of low-temperature grains is needed. In the model by Waters et al. (1989) the distribution extended out to $2500 R_{\star}$ (corresponding to $500 \mathrm{AU}$ ) in order to account for the $60 \mu \mathrm{m}$ flux. Since there is now evidence that the spectrum continues as a blackbody down to at least $850 \mu \mathrm{m}$, the required distribution would have to extend to temperatures as low $3 \mathrm{~K}$, while the interstellar radiation field alone already heats dust to considerably higher temperatures. We will therefore not investigate such a fine-tuned distribution of small grains in a spherical shell any further.

There are two remaining possibilities to explain the blackbody shape of the IR-excess. The first possibility would be that the individual grains that are responsible for the continuum emission are radiating like a blackbody themselves. In that case, the grains must be large compared to all wavelengths present in the SED, and the temperature distribution relatively narrow. The second possibility is that the individual grains are not blackbody emitters; in that case we propose that the blackbody radiation is produced by an optically thick disk surface which radiates only in a narrow temperature range. In the remainder of the paper, we will investigate these two possibilities and their consequences.

Assuming that the IR emission is produced by a disk with height $\mathrm{H}$ above the midplane (total thickness of the disk is $2 \mathrm{H}$ ), the dimensions can be derived from the constraint that one third of the stellar luminosity should be absorbed by the disk. If the disk is infinitely optically thick to the stellar radiation (like a solid surface) and located at a distance $R$ from the star, then the disk surface is $2 \pi R \cdot 2 H$; this surface must then cover a solid angle of $\frac{1}{3} \times 4 \pi R^{2}$, so we find for the height of the disk

$\frac{H}{R}=\frac{1}{3}$.

This is actually a lower limit for the height. If the disk is not optically thick but absorbs on average only a fraction $1-e^{-\tau}$ of the stellar radiation, the height would have to be larger by a factor $1 /\left(1-e^{-\tau}\right)$.

$\frac{H}{R}=\frac{1}{3} \frac{1}{1-e^{-\tau}}$

As the only assumption that went into Eqs. (7) and (8) is that the dust is in a disk, the limit on the height $H$ is a major constraint on any disk model. 


\section{Narrow temperature distribution of blackbody grains}

We will now investigate the possibility that the blackbody shape of the continuum is due to a collection of "large" grains that each radiate like a blackbody. "Large" grains in this case means that the size of these grains must be larger than all wavelengths present in the SED; therefore the grains must be at least mm-sized.

\subsection{Required disk structure}

Using the equation of thermal equilibrium of dust grains, we can translate the limits imposed on the temperature from Eqs. (5) and (6) into limits on the distance of the dust grains from the central star :

$T_{\mathrm{d}}=\left(\frac{1}{2} \frac{R_{*}}{R}\right)^{1 / 2} T_{*}$.

Using a stellar effective temperature $T_{\star}$ of $7500 \mathrm{~K}$ and an effective radius $R_{\star}$ of $47 R_{\odot}$ (Bakker et al. 1998), we find that the dust grains must be between 3.5 and 8 AU from the star.

There are a few caveats on these distance limits. Since these numbers have been calculated assuming that each dust grains receives the unattenuated radiation from the star, the outer radius is an upper limit. If we take into account that a fraction of the stellar radiation gets absorbed, it can be shown that the outer distance decreases for small optical depth with a factor of $e^{-\tau / 2}$; assuming an optical depth of $\tau=0.5$, the outer limit would therefore go down to 6.2 AU. According to Eq. (8) the same optical depth would require the disk height to reach $2 H=1.7 R$. In this way, the radial extent of the disk can be traded against its height. For very low optical depths, the disk must be extremely high. For modest optical depths, the disk height decreases towards the limit given in Eq. (7), but the radial extent must be smaller.

We can also estimate the disk mass. The total absorbing surface is $A_{\mathrm{abs}}=2 \pi R \cdot 2 H$ which must be equal to the cross section of $N$ grains: $2 \pi R \cdot 2 H=N \pi a^{2}$. The total dust mass is then

$M_{\text {dust }}=2.85 \times 10^{-6} M_{\odot} \frac{R}{4 \mathrm{AU}} \frac{H}{1.4 \mathrm{AU}} \frac{\rho_{\text {dust }}}{2.7 \mathrm{~g} \mathrm{~cm}^{-3}} \frac{a}{1 \mathrm{~mm}}$.

It is clear that the major difficulty of the model with large grains in a narrow temperature range is how to realize the very particular arrangement needed;

1. The disk needs to have a fine-tuned radial optical depth between one and two over all scale heights. Optical depths less than one will fail to absorb enough stellar radiation. Optical depths larger than two will produce a temperature range which is too wide.

2. A disk is required which is more extended in the vertical then in the radial direction.

The scale height of such a disk could be provided either by a gas-rich disk with a hydrostatic distribution of gas in which the grains are suspended, or dynamically in a gas poor disk. We will discuss both scenarios.

\subsection{Dust grains in a gas-rich disk}

If the disk were gas-rich, the same hydrostatic pressure which is required to hold up the vertical height would also act in the radial direction, attempting to spread the disk in this direction. Since in a Keplerian disk the radial component of the gravitational force is balanced by the centrifugal force, stability of the disk would also require significant sub-Keplerian rotation in the outer parts of the disk in order to balance the large gas pressure gradient in the disk. The resulting shear motion leads to transport of angular momentum which would again spread out the disk in radial direction. Another concern is grain settling. The settling time for grains is a gas-rich disk is given by Eq. (26). If we scale the dust mass of $2.85 \times 10^{-6} M_{\odot}$ with a nominal gas-to-dust ratio of 100 , we can estimate a gas mass in the disk of $2.85 \times 10^{-4} M_{\odot}$. Using 3.5 AU and 6.2 AU for the radial extent of the disk, we find an average surface density of $30 \mathrm{~g} / \mathrm{cm}^{2}$. At $4 \mathrm{AU}$, this corresponds to a settling time of $150 \mathrm{yr}$ for $1 \mathrm{~mm}$ dust grains. Grain settling would half the disk height in this time scale. This estimate is independent of the grain size since the settling time depends on the ratio of surface density over grain radius which is constant for the above estimate. A large height could be kept for a longer time only if the gas-todust ratio in the disk would be much greater than 100 .

It is therefore highly questionable if a cloud of large dust grains supported by a hydrostatic gas-rich disk could reach a stable configuration in accordance with the observational constraints.

\subsection{Dust grains in a gas-poor disk}

The other possibility is that the disk is gas-poor. It would then be a structure resembling the asteroid belt or the Kuiper Belt in the solar system, or the debris disks found around mainsequence stars (e.g. Backman \& Paresce 1993; Chen \& Jura 2001). In such disks dust grains are produced by collisions between larger bodies. Since grains smaller than about $1 \mathrm{~mm}$ would be blown away by radiation pressure, this would also lead to a natural explanation for the absence of small grains indicated by the blackbody spectrum. In a gas-poor disk, the dust grains move on Keplerian orbits around the central star. The vertical structure of such a disk is then given by the distribution of orbital inclinations. One could imagine that the radial extent of such a disk is set by a sheparding planet, much like it is in Saturns rings.

However, it is easy to show that such a disk cannot keep the required large inclination distribution. As we have discussed above, the disk must have an optical depth of order one in the radial direction. Since the disk is higher than its radial extent, this immediately implies that the vertical optical depth will be of order one or even larger. Therefore, a dust grain moving on an inclined orbit will experience at least two collisions during each orbit. This is simply due to the fact that both the collision cross section and the absorption cross section are set by the geometrical cross section of the grains.

The collisions between grains will either destroy the grains if the velocity is larger than a few $\mathrm{km} \mathrm{s}^{-1}$ (Tielens et al. 1994), or dissipate energy. The energy dissipation leads to a rapid 
flattening of the disk. Jeffreys (1947) showed for the case of Saturns rings that such a disk quickly collapses to a mono layer thickness. A more detailed study including the effects of gravitational stirring of bodies embedded in the disk (Cuzzi et al. 1979) showed that the thickness is given by a few times the diameter of the largest bodies in the disk. To counteract the collapse of the disk, massive external stirring would be needed, which would spread the disk in vertical and radial direction, in contradiction to the observed narrow range of temperatures.

An interesting analogy for this model is the star HD 98800 which was described by Zuckerman \& Becklin (1993). This star is a binary system composed of two pre-main-sequence $\mathrm{K}$ dwarfs. The dust disk, located around component B, absorbs about $20 \%$ of the radiation of this component. It is easy to see that the large scale height required cannot be due to hydrostatic equilibrium (see Sect. 6.1) since the stars are not luminous enough. Therefore the most likely explanation for this system is that it is indeed a debris system. However, the same arguments limiting the lifetime of such a system apply (Zuckerman \& Becklin 1993). Possibly, the disk in this system has been produced by a recent destructive event, or is maintained by the interactions of the binary system.

\section{An optically thick disk: The wall model}

The second explanation for the blackbody spectrum of HR 4049 is that the emission comes from an optically thick disk surface.

The scenario which we have in mind is a highly optically thick cylindrical (see Fig. 12) circumbinary dust disk which is illuminated from inside by the central star. The high optical depth prevents radiation to penetrate deeply into the disk which is therefore cold and invisible against the bright inner rim. What we observe as the blackbody radiation is the part of the inner disk rim which is directly visible to the observer.

The disk will have a very hot inner rim, which will be at a single temperature if the rim is concave (i.e. at constant distance from the star). A vertical or more convex inner rim will produce a spectrum that is a bit more multi-temperature, but this deviation may still lie within the temperature constraints derived in Sect. 3.2.

The optical depth of this disk must be high, at all wavelengths considered. In fact, the optical depth must be so high that the colder dust which is not directly illuminated by the star does not contribute to the emergent spectrum. We will quantify this below.

In the optically thick model, the constraints on the particle size and on the radial extent which we derived in Sect. 4 can be relaxed. The individual grains do not have to be large, and there is no limit on the radial extent.

\subsection{Geometrical constraints revisited}

In Sect. 3.3 we have shown that the height of an optically thick disk must be $H=\frac{1}{3} R$ in order to absorb sufficient radiation, which was derived from the ratio of IR and stellar luminosities.

We envision the emitting surface to be optically thick and radiating only into a half-space (see Fig. 12). The flux seen by an observer will depend upon the viewing angle under which the system is seen. From a nearly pole-on direction, the projected surface would be small and the IR radiation received low. From a nearly edge-on direction, the projected surface is large and the observed flux high. The observed IR luminosity therefore roughly scales with $\sin i$. Since the $H / R$ ratio depends on the emitted IR luminosity rather than the observed luminosity, there is an uncertainty in the real value of $H / R$, depending on the inclination at which we observe the system. Because of the significant variations in circumstellar extinction during the orbital motion of HR 4049, a larger (near edge-on) inclination seems more likely, meaning that the real $H / R$ could be somewhat smaller than $\frac{1}{3}$.

From the temperature of the dust one can easily compute the distance of the inner rim to the central star. This distance is a factor of two larger than the distance of black body grains in an optically thin environment because the surface can cool only into a solid angle of $\pi$ instead of $4 \pi$ in the optically thin case. If this rim is located at a distance $R$ from the star, then the stellar flux it receives is: $F_{*}=\left(R_{*} / R\right)^{2} \sigma T_{*}^{4}$. The flux emitted by the wall is $F_{\text {wall }}=\sigma T^{4}$. Therefore the temperature of the wall, if located at a distance $R$, is $T=\left(R_{*} / R\right)^{1 / 2} T_{*}$. For $T=$ $1200 \mathrm{~K}, T_{*}=7500 \mathrm{~K}$ and $R_{*}=47 R_{\odot}$ one obtains a radius of the inner surface of $8.5 \mathrm{AU}$. If one considers the fact that the wall is also irradiating itself, then one must take into account that the total irradiation flux of the wall will be about a factor of $1+\Omega / 4 \pi$ higher than the direct stellar irradiation. By taking $\Omega / 4 \pi \simeq 1 / 3$, as derived above, one finds $R=\sqrt{4 / 3} \times 8.5 \mathrm{AU}=$ 9.9 AU. It should be noted that the estimate of $1+\Omega / 4 \pi$ as the enhancement factor for the irradiation is very rough, but suffices for now. For a detailed discussion, see Dullemond et al. (2001).

\subsection{Emission from an irradiated dust wall}

We now turn to the question of whether an irradiated wall will indeed produce an emission that is sufficiently close to a blackbody curve to fit the SED of HR 4049. Since 2D radiative transfer calculations with full frequency dependence and at optical depths reaching a million are very cumbersome, we will tackle this problem in a two-step approach: first we will use a 1-D radiative transfer calculation in order to see if the inner surface of the wall does indeed produce blackbody-like emission. Then we will use a 2-D diffusion calculation in order to see if the emission of the rest of the disk (surface and outer rim) can be sufficiently suppressed using high optical depth.

For the 1-D calculation, we consider infinite half-space filled with dust: for $x<0$ there is vacuum, for $x>0$ there is a constant density medium of dust grains. This half-space is illuminated (on the $x<0$ side) with a flux $F_{v}^{*}$ which is taken to be the stellar flux as observed at a radius of 8.5 AU (we ignore the effects of self-irradiation mentioned above). We run the calculation for a mixture of grains of two sizes: small $(0.1 \mu \mathrm{m})$ grains, and large grains. We take the opacity of Draine \& Lee (1984) for small grains, and a simple grey opacity for our "large" grains. The grey opacity guarantees that the optical depth will be large at all wavelengths, a very important 


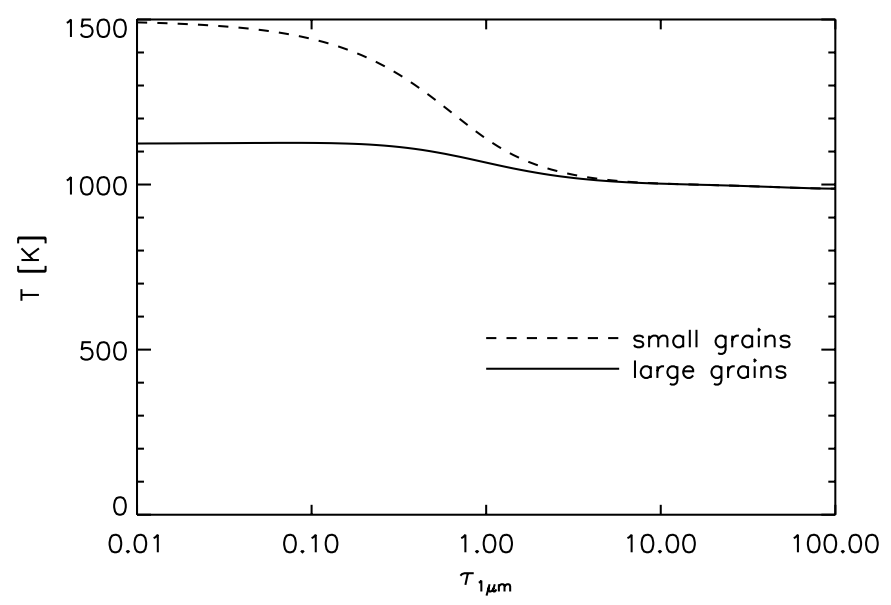

Fig. 4. Temperature structure of the 1D calculation.

requirement of the wall model. We include the small grains in order to see if the superheating of small grains close to the surface of the disk will cause significant deviations from the blackbody shape. The requirement of large optical depth at all wavelengths could also be met with non-grey, small-grain opacities. In this case the limiting criterion would be that the optical depth at $850 \mu \mathrm{m}$ must be large. The transfer is performed using a frequency-dependent discrete-ordinate method (i.e. ray-by-ray transfer at each frequency bin and for each angle). The transfer equation is:

$\mu \frac{\mathrm{d} I_{\mu, v}}{\mathrm{~d} x}=\sum_{i} \rho_{i} \kappa_{v}^{i}\left[B_{v}\left(T_{i}\right)-I_{\mu, v}\right]$

where $\kappa_{v}^{1}$ and $\kappa_{v}^{2}$ are the opacities of the small grains and the large grains respectively, and idem for the densities $\rho_{1}$ and $\rho_{2}$ and the temperatures $T_{1}$ and $T_{2}$. We ignore scattering for simplicity. The symbol $\mu$ is the cosine of the angle of the radiation. At every point $x$ in the slab, the temperature of the dust grains is determined by the radiative equilibrium equation:

$\int_{0}^{\infty} \kappa_{v} B_{v}\left(T_{i}\right) \mathrm{d} v=\int_{0}^{\infty} \kappa_{v} J_{v} \mathrm{~d} v$

where the mean intensity $J_{v}$ is given by

$J_{v}=\frac{1}{2} \int_{-1}^{1} I_{\mu, v} \mathrm{~d} \mu$.

Using Lambda Iteration with $\mathrm{Ng}$ acceleration we iterate this set of equations to convergence and find the solutions shown in Fig. 4.

One sees that for the large grains, the temperature remains almost constant as a function of optical depth. The small grains superheat on the disk surface. At higher optical depth, the two temperatures become the same because at large optical depth both grain species become radiatively coupled. By performing direct ray-tracing through this model atmosphere, we can obtain the predicted SEDs, which are shown in Fig. 5. The emerging spectra are close to pure blackbody curves, in fact the fat curve is indistinguishable from a blackbody. The superheated small grains produce a slight broadening of the SED towards

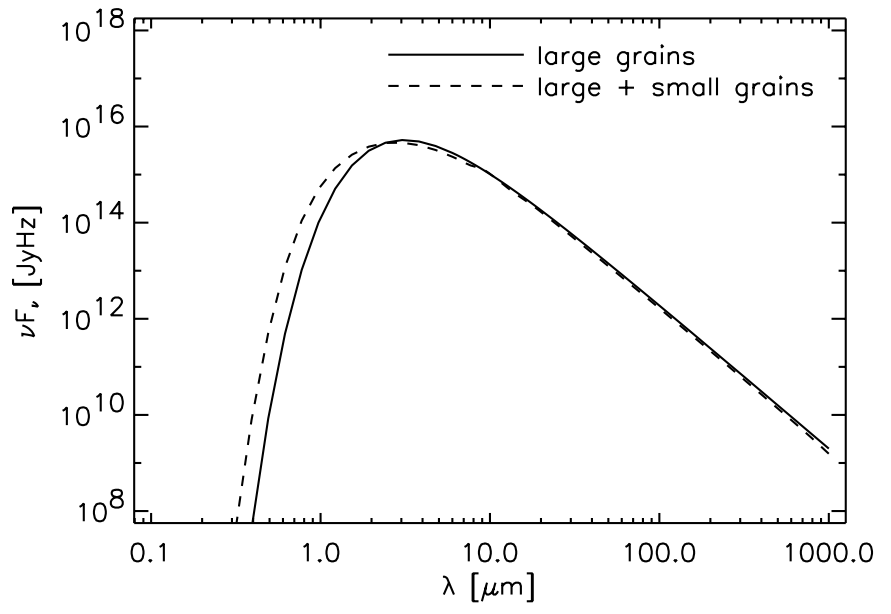

Fig. 5. The SED produced by the 1D calculation. The solid curve is indistinguishable from a single temperature blackbody.

short wavelength. We conclude that an irradiated high optical depth surface indeed radiates like a blackbody and could in principle be used to explain the SED of HR 4049.

\subsection{Diffusion though the disk}

In order to address the issue of diffusion through the disk towards the surface and towards the outer edge, we need to perform a 2-D radiative transfer calculation. Unfortunately, realistic radiative transfer calculations in 2-D are rather cumbersome and complex, in particular at the very high optical depths needed for our disk model. Under these circumstances, a reasonably reliable approximation of the 2-D transfer problem can be made using the equations of radiative diffusion.

Since we are interested in the qualitative behavior of the radiative diffusion through the disk, we keep the disk geometry highly simplified. We assume a constant density torus limited in radius by $9 \mathrm{AU}<R<18 \mathrm{AU}$ and in $\Theta$ (the angle from the pole) between $\pi / 2-1 / 3<\Theta<\pi / 2+1 / 3$ - consistent with the $H / R=1 / 3$ requirement. We compute first how the direct stellar radiation penetrates the inner few mean free paths of the inner edge:

$F_{v}^{*}(R, \Theta)=\frac{L_{v}^{*}}{4 \pi R^{2}} \exp \left\{-\int_{R_{\text {in }}}^{R} \rho \kappa_{\nu} \mathrm{d} R\right\}$.

We now assume that the absorbed radiation is re-emitted as IR radiation. This diffuse IR radiation is treated as a separate radiation field. We do this in a frequency-averaged way, and write down the diffusion equation for the frequency-averaged mean intensity $J(R, \Theta)$ :

$\frac{1}{R^{2}} \frac{\partial}{\partial R}\left(\frac{R^{2}}{3 \rho \kappa} \frac{\partial J}{\partial R}\right)+\frac{1}{R \sin \Theta} \frac{\partial}{\partial \Theta}\left(\frac{\sin \Theta}{3 R \rho \kappa} \frac{\partial J}{\partial \Theta}\right)=-S$

where $\kappa$ is the Rosseland mean opacity. This equation describes the radiative diffusion in polar coordinates, derived from the first two moment equations with Eddington closure. We also implicitly assume (for simplicity) that the mean opacity does not change very much from location to location, since otherwise additional terms have to be included in the diffusion equation. 


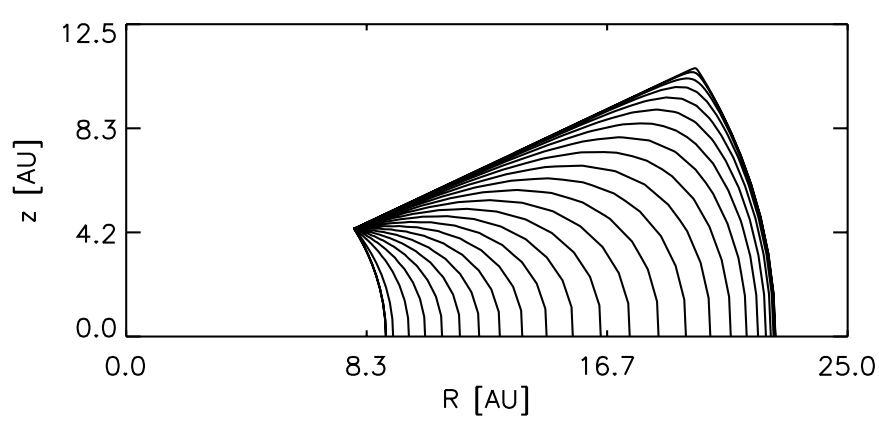

Fig. 6. Temperature contours of the diffusive disk. The temperature at the inner boundary is $1200 \mathrm{~K}$, further contours are in steps of $50 \mathrm{~K}$.

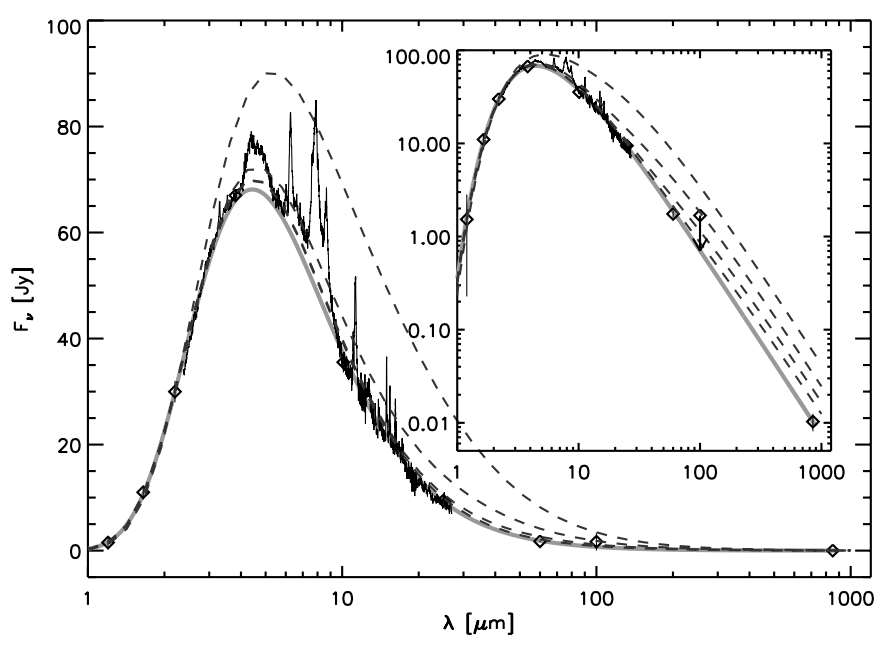

Fig. 7. Fits to the observed SED with diffusion calculations for tori with different optical depth. The solid grey line shows the $1150 \mathrm{~K}$ blackbody fit. The dashed lines are (from top to bottom) for optical depth $10^{2}, 10^{3}, 10^{4}$, and $10^{5}$.

The source term $S$ is given by:

$S=\frac{1}{4 \pi} \int_{0}^{\infty} \rho \kappa_{v} F_{v}^{*} \mathrm{~d} v$

This source term describes the transition of energy from primary stellar radiation into reprocessed diffuse infrared radiation. Since all primary radiation is absorbed in the innermost few mean free pathlengths, this source term will be only nonnegligible close to $R=R_{\mathrm{in}}$. So for larger radii the above diffusion equation (Eq. (15)) effectively states that the divergence of the flux should be zero, i.e. that energy is conserved. The boundary conditions at all edges are taken to be those described in Rybicki \& Lightman (1979).

The 2-D diffusion equation can now be solved by writing it into a matrix equation, and solving this using standard techniques such as the biconjugate gradient method (e.g. Numerical Recipes 1992). Once we find $J(R, \Theta)$, we can compute the dust temperatures from:

$\frac{\sigma}{\pi} T(R, \Theta)^{4}=J(R, \Theta)+\frac{S}{\rho \kappa_{\mathrm{P}}(T)}$

where $\kappa_{\mathrm{P}}(T)$ is the Planck mean opacity at the dust temperature.

We run several models, with an equatorial Rosseland optical depth between $\tau=100$ and $\tau=10^{5}$ and one for $\tau=10^{6}$.
The resulting temperature profile for the $\tau=10^{4}$ case is shown in Fig. 6. In Fig. 7 we show the SEDs of these tori, as seen from an inclination of 45 degrees. We used a 2-D ray-tracing utility to produce these SEDs from the temperature distribution resulting from the 2-D diffusion equations.

One sees that even for very high optical depth the spectrum will exceed a pure blackbody curve at long wavelengths. The $\chi_{v}^{2}$ for the $\tau=10^{5}$ fit is 2.85 . For high optical depths only a very tiny amount of radiation is leaking through the disk. But since the Rayleigh-Jeans slope of a blackbody curve does not contain much energy, even this tiny amount of leakage shows up at long wavelengths. These results are in agreement with the simple estimate that the flux at the surface and the outer edge of the torus is proportional to $1 / \tau$, where $\tau$ is the optical depth towards the inner edge. If we assume that the flux is proportional to $T^{4}$, then one can estimate that the temperature of the outer- and upper/lower surfaces of the torus is proportional to $T_{\text {surf }} \propto \tau^{-1 / 4}$. This means that in order to suppress the temperature of these back-surfaces of the disk by a factor of 0.1 one must have an optical depth of $\tau \simeq 10^{4}$. A pure blackbody emission from this torus model therefore requires very high optical depth.

\section{Disk properties}

The disk we are proposing to be the source of the $1150 \mathrm{~K}$ blackbody radiation in HR 4049 certainly is unusual, because of the high scale height (covering fraction) at the inner boundary and the high optical depth. In this section, we will derive properties of the disk including mass estimates and stability considerations in order judge how realistic such a model can be. We will therefore investigate the following questions.

1. Can we understand the large disk height $(H / R=1 / 3)$ ?

2. Can we place limits on the grain size?

3. What disk mass is required to make the disk sufficiently optically thick?

4. How long will it take for the dust grains to settle towards the midplane?

5. Will the disk remain radially mixed, or will the radiation pressure of the star lead to a grain-gas separation?

6. Will radiation pressure of the star be sufficient to blow away (parts of) the disk?

As we already discussed in Sect. 4.3, a gas-poor disk cannot keep the required scale height because of frequent collisions. This argument is even much stronger for an optically thick disk. We will therefore assume the disk is gas-rich and in hydrostatic equilibrium, to which we can apply the standard theory of hydrostatic disks. We will adopt a cylindrical axisymmetric coordinate system with radial coordinate $r$ and vertical coordinate $z$. We will often refer to scale heights in the following sections; we remind the reader that these scale heights correspond to the vertical coordinate starting from the equatorial plane. For the following estimates we will for simplicity assume that the disk has a constant surface density $\Sigma_{0}$ and a constant scale height $h_{0}$ (i.e. a non-flaring disk, see below) in a distance range $\Delta r=r_{1}-r_{0}$ from the star. We will further assume that inside the disk the dust-to-gas ratio is $f_{\mathrm{d}}=0.01$ and that the vertical 
distribution of the gas is given by hydrostatic equilibrium in an isothermal disk. We assume the dust particles all to have the same size $a$ and a specific density $\rho_{\mathrm{d}}$.

Armed with these properties, we may calculate the total disk mass

$M_{\text {disk }}=2 \pi\left(r_{1}^{2}-r_{0}^{2}\right) \Sigma_{0}$.

When evaluating equations numerically in the following, we will always use a standard model of the disk which contains $0.3 M_{\odot}$, with $r_{0}=10 \mathrm{AU}$ and $r_{1}=20 \mathrm{AU}$. This corresponds to a surface density of $1414 \mathrm{~g} \mathrm{~cm}^{-3}$. The disk mass of $0.3 M_{\odot}$ is very high and should be considered an upper limit for the possible disk mass since significantly higher masses will lead to gravitational instabilities if $M_{\text {disk }}>\frac{H}{R} M_{\star}$ (Pringle 1981). We use this high mass in order to maximize the stability of the disk against grain settling or radiation pressure.

\subsection{Vertical scale height from hydrostatic equilibrium}

We assume the disk to be gas-rich. Its height can therefore be calculated from the assumption of hydrostatic equilibrium. The disk pressure scale height $h_{\mathrm{p}}$ is given by the square root of the ratio of the sound speed and the local Kepler frequency and can be written as (e.g. Chiang \& Goldreich 1997)

$$
\frac{h_{\mathrm{p}}}{r}=\chi \sqrt{\frac{k T r}{G\left(M_{1}+M_{2}\right) \mu m_{\mathrm{p}}}}
$$

where $M_{1}$ and $M_{2}$ are the masses of the two stars in the HR 4049 system, $\mu$ is the mean molecular weight and $m_{\mathrm{p}}$ is the proton mass. It should be noticed that this formula holds for an annulus of a thin disk, and may be inaccurate for the inner edge of a thick disk. For $r=8.6 \mathrm{AU}, M_{1} \simeq 0.56 M_{\odot}$ and $M_{2} \simeq M_{1}$, and $T=1150 \mathrm{~K}$ this amounts to

$\frac{h_{\mathrm{p}}}{r}=0.19$.

If we compare this computed scale height with the observed required scale height (see Sect. 3.3), which was $H / r=1 / 3$, and if we take into account that the surface height (the height at which $\tau=1$ ) is usually a few times the pressure scale height we arrive at

$$
\frac{H}{r}=0.19 \frac{H}{h_{\mathrm{p}}}
$$

where $H / h_{\mathrm{p}}$ is roughly in between 1 and 4 for a Gaussian vertical density distribution (Chiang \& Goldreich 1997)

Therefore, a hydrostatic disk can reach the required height without any difficulty. In fact, a mechanism to truncate the disk somewhere between one and two pressure scale heights seems to be required in order to match the observational constrains.

In the passive disks around young stars, the surface irradiation of thin circumstellar disks leads to an increase of the ratio $h_{\mathrm{p}} / r$ with distance from the star. Such disks are called flaring disks, and have been successfully used to explain the flat SEDs of young stars with disks (Kenyon \& Hartmann 1987; Chiang \& Goldreich 1997). Could the disk around HR 4049 also be flaring? The blackbody shape of the SED is clear evidence that

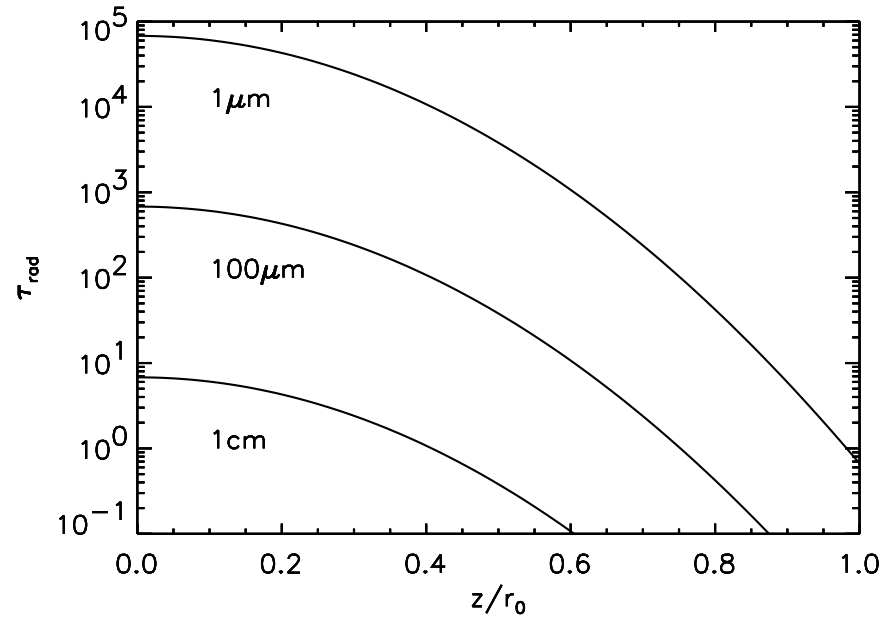

Fig. 8. The optical depth for short wavelength radiation along radial rays through the torus as a function of height. The different curves are for different grains sizes with fixed total mass of $2 M_{\odot}$.

this is not the case, because flaring disks generally have very broad, multicomponent SEDs. The physical criterion for flaring to occure is that the disk midplane temperature must decrease more slowly than $r^{-1}$, so that $h_{\mathrm{p}} / r$ increases with distance (see Eq. (19)). In the wall model, the disk temperature is not determined by the radiation reaching the disk surface. Instead, radiation is only impacting on the inner rim and transported outwards through the disk by diffusion. The temperature will therefore decrease quickly with distance from the star, and $h_{\mathrm{p}} / r$ will decrease, preventing any flaring. The shape of such a diffuse disk inner rim has recently been discussed in detail in connection with the near IR emission of Herbig AeBe stars (Dullemond et al. 2001).

\subsection{Radial optical depth}

The density structure $\rho(z)$ in the disk assuming hydrostatic equilibrium is given by (Chiang \& Goldreich 1997)

$\rho(z)=\frac{\Sigma_{0}}{\sqrt{2 \pi} h_{0}} \exp \left\{-\frac{z^{2}}{2 h_{0}^{2}}\right\}$.

If dust and gas are perfectly mixed, the number density of dust grains is given by

$n_{\mathrm{d}}(z)=\frac{f_{\mathrm{d}} \rho(z)}{m_{\mathrm{d}}}=\frac{3 f_{\mathrm{d}} \rho(z)}{4 \pi a^{3} \rho_{\mathrm{d}}}$

where $f_{\mathrm{d}}$ is the dust-to-gas ratio and $m_{\mathrm{d}}$ is the mass of a single dust grain. The dust opacity is then given by

$c_{\mathrm{d}}(z)=n_{\mathrm{d}} Q(a, \lambda) \pi a^{2}=\frac{3 f_{\mathrm{d}} \rho(z) Q(a, \lambda)}{4 a \rho_{\mathrm{d}}}$

where $Q(a, \lambda)$ is the absorption efficiency factor. To calculate the radial optical depth we use the assumption, that the radial extent of the disk is small compared to the distance of the inner rim to the star, so that we can consider the ray going through 


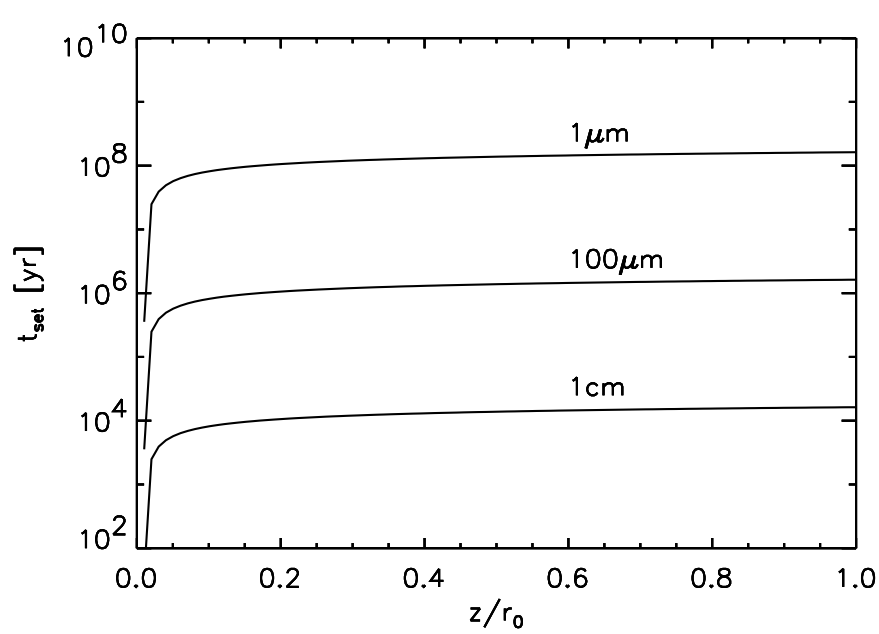

Fig. 9. Settling times for dust grains of different size.

the disk material on a constant height $z$. The optical depth on such a ray is given by

$\tau(z, \lambda)=c_{\mathrm{d}} \Delta r=\frac{3 f_{\mathrm{d}} \Delta r \Sigma_{0} Q(a, \lambda)}{4 \rho_{\mathrm{d}} a \sqrt{2 \pi} h_{0}} \exp \left\{-\frac{z^{2}}{2 h_{0}^{2}}\right\}$.

In Fig. 8 we show the optical depth for our standard disk as a function of height $z / r_{0}$ in the disk. Since we keep the total disk mass constant, the optical depth is different for different grain sizes. What we have plotted is the optical depth at very short wavelength, using a geometrical cross section for the grains. This number is an estimate for the optical depth at all wavelengths smaller than $2 \pi a$. For interstellar grain sizes $(0.1 \mu \mathrm{m})$, very high optical depths of up to $10^{6}$ can be reached, but only at short wavelengths. The SED measurement with the longest wavelengths is the $850 \mu \mathrm{m}$ SCUBA measurement, and the $100 \mu \mathrm{m}$ grains show a better estimate for the optical depth at these wavelength. Here the standard disk model can reach $\tau=10^{3}$, and with a disk mass of $2 M_{\odot}, \tau=10^{4}$ might be feasible. As shown in Fig. 7, this optical depth gives a reasonable fit to the observed SED even though the flux at $60 \mu \mathrm{m}$ and $850 \mu \mathrm{m}$ is still slightly over predicted. We therefore conclude that a massive disk can indeed reach very high optical depth. But we also find that the grains should not be much bigger than $100 \mu \mathrm{m}$, or the optical depth will become too low.

\subsection{Grain settling}

In order to reach the required covering faction, the optical depth of the disk must be high not only in the midplane, but also at higher $\mathrm{z}$, up to $z / r_{0} \approx 0.3$. Since the disk opacity is entirely due to dust grains, but the disk scale height is entirely due to gas pressure, gas and dust have to be well mixed. From protoplanetary accretion disks it is known that dust grains will eventually start to settle towards the midplane. The time for a dust grain to settle from height $z$ to height $z_{0}$ in a quiescent hydrostatic disk is given by Miyake \& Nakagawa (1995)

$t_{\mathrm{set}}=\frac{\pi}{2} \frac{\Sigma_{0}}{\rho_{\mathrm{d}} a} \frac{1}{\Omega_{\mathrm{k}}} \ln \frac{z}{z_{0}}$

where $\Omega_{\mathrm{k}}=\sqrt{G M / r^{3}}$ is the Kepler frequency in the disk.

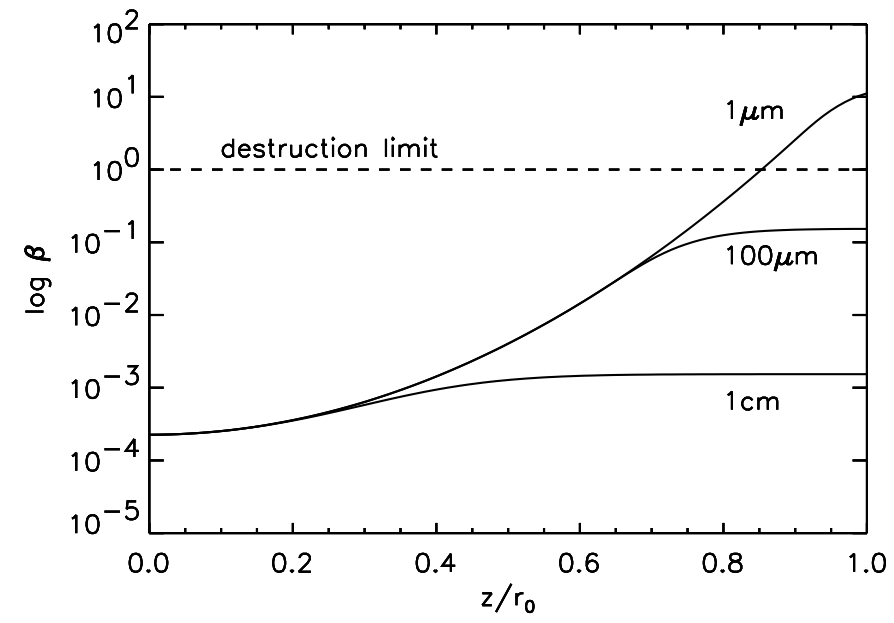

Fig. 10. Ratio of total radiation pressure on a radial column of material over the gravitational attraction from the binary. Different curves are for different grain sizes with a fixed total mass of $0.3 M_{\odot}$. The dashed line marks the blowout limit.

Figure 9 shows the settling times for different grain sizes in the standard disk. The timescale varies from $10^{8}$ years for $1 \mu \mathrm{m}$ grains to $10^{4}$ years for $1 \mathrm{~cm}$ grains. Therefore, at least for grains smaller than about $100 \mu \mathrm{m}$, grain settling does not provide a serious constrain to the disk model. However, for grains larger than a few $\mathrm{cm}$, the lifetime of the disk becomes so short that we would be lucky to see HR 4049 in this stage at all.

\subsection{Radiation pressure and disk stability}

To estimate at what height the radiation pressure will actually be large enough to blow away the entire disk (gas and dust), we make the following calculation. We consider a radial "beam" of material in the disk. The beam is all the material outside a surface element $\Delta S$ in the disk wall. The radiation pressure available for that beam of material is given by the momentum flux of all the radiation entering $\Delta S$ and being absorbed in the beam, thus $L_{\star} \Delta S\left(1-e^{-\tau}\right) / 4 \pi r_{0}^{2} c$. In order to blow away a part of the disk, the radiation pressure must be strong enough to lift the entire mass outside of the surface element of the rim. This mass is given by $\rho(z) \Delta r \Delta S$. The ratio of radiative and gravitational force is then

$\beta=\frac{L_{\star}\left(1-e^{-\tau}\right)}{4 \pi G M c \rho(z) \Delta r}$,

and $\beta=1$ marks the limit where the radiation force can remove the entire beam of material. Inserting the expression for the density Eq. (22) and solving for $z$ under the assumption $\tau \gg 1$, we find that the critical height $z_{\text {crit }}$ where the disk is blown away by the stellar radiation is given by

$z_{\text {crit }}^{2}=-2 h_{0}^{2} \ln \left[\frac{L_{\star}}{4 \pi c} \frac{1}{G M} \frac{\sqrt{2 \pi} h_{0}}{\Sigma_{0} \Delta r}\right]$.

Figure 10 shows the value of $\beta$ for the standard disk and 3 different grain sizes as a function of height in the disk. The positions where the curves cross the line $\beta=1$ is the location of $z_{\text {crit }}$. We can see that for grains larger than $\approx 10 \mu \mathrm{m}$, the radiation 


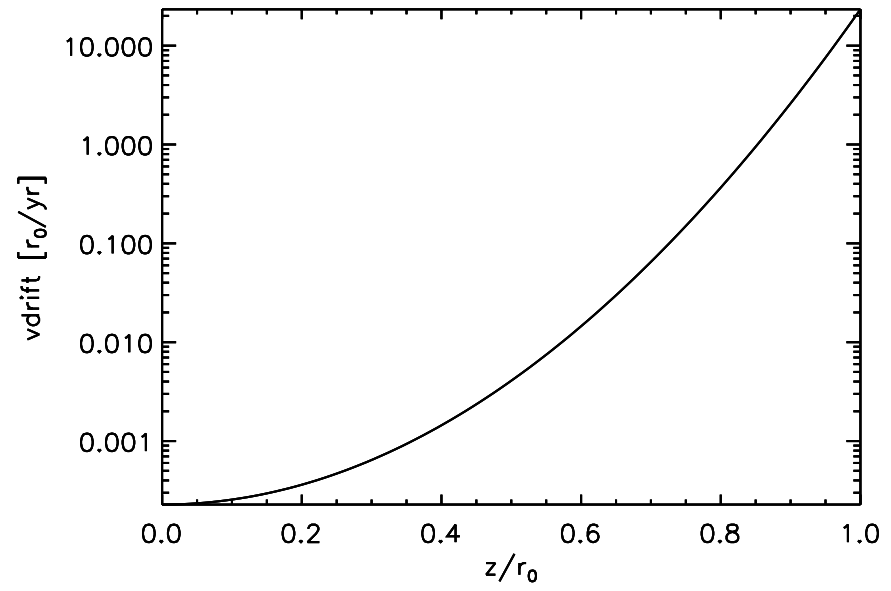

Fig. 11. Equilibrium drift velocity of grains assuming direct exposure to unattenuated stellar light. Note that the drift velocity is given in units $r_{0}$ per year.

pressure is never large enough to remove the disk (gas + dust). For low height this is due to the large mass in a radial beam of material. For large height, the disk becomes optically thin, and an insufficient fraction of the radiation is absorbed in the disk. Only if the dust in the disk consists mainly of grains smaller than $10 \mu \mathrm{m}$, the parts of the disk with $z / r_{0}>0.8$ can be completely removed by radiation pressure.

\subsection{Grain drift}

Removing a part of the disk is not the only effect radiation pressure on dust grains can have. Since the radiation pressure only acts on the dust grains, the grains can be pushed outwards through the gaseous disk. In parts of the disk which are optically thick, all but the innermost grains are shielded from direct stellar radiation. The internal radiation field in the disk is almost perfectly isotropic, and the radiation force and corresponding drift motion will be small. However, in the optically thin parts of the disk and also at the inner rim, grains do receive the full stellar radiation. In these regions, the velocity at which the grains move can be calculated by assuming an equilibrium between the radiation pressure on a dust grain and the drag force due to the motion of the grain. In the case of subsonic drift, the equilibrium velocity is given by (e.g. Dominik et al. 1989)

$v_{\mathrm{dr}}=\frac{L}{4 \pi r^{2} c} \frac{Q}{\Omega_{\mathrm{k}}} \frac{c_{\mathrm{s}}}{v_{\mathrm{th}}} \frac{\sqrt{2 \pi}}{\Sigma_{0}} \exp \left\{\frac{z^{2}}{2 H^{2}}\right\}$.

In Fig. 11 we have plotted the drift velocity as a function of height in the disk. In the disk midplane, the drift velocity is about $10^{-4} r_{0} /$ yr. Grains can therefore be pushed into the disk on a timescale of $10^{4}$ years only. This defines the tightest constraint on the life time of the disk. Note, however, that this limit was calculated under the assumption of a quiescent disk. Turbulent motions near the inner disk boundary could be efficient in keeping the grains close to the inner boundary. At larger height in the disk, the drift becomes very efficient. At $z / r_{0}=1 / 3$, grains can be pushed through the disk in less than
$10^{3}$ years. This provides an effective truncation mechanism for the disk height and may explain the difference between the calculated hydrostatic height of the gas disk and the observed covering fraction.

\section{Discussion}

The circumstellar environment of HR 4049 is certainly unusual in many ways. There are three major constraints which are given by the observations, and which must be explained by any model:

1. The UV extinction is highly variable, non-grey and in phase with the orbital motion.

2. The IR excess contains $1 / 3$ of the stellar luminosity.

3. The spectral shape of the excess is a perfect blackbody from $1 \mu \mathrm{m}$ to $850 \mu \mathrm{m}$.

From (1) one can immediately conclude that the distribution of material is not spherical. Our line-of-sight passes through a region with a strong gradient in extinction, probably the upper part of a disk-like structure. The grains causing the extinction must be small since large grains would imply grey extinction. If a disk-like geometry is causing the reprocessing of stellar light, (2) implies that the disk must be at least marginally optically thick and that it must have a large height. The most difficult part turns out to be explaining (3). We have presented two different scenarios on how a disk may produce a blackbody emission: (i) large grains in a marginally optically thick disk with very small radial extent and (ii) grains of unknown size in a disk with very high optical depth. In this discussion we will highlight the virtues and the shortcomings of both models.

\subsection{Large grains versus small grains}

If the blackbody shape of the IR excess is caused by thermal emission of large grains (see Sect. 4), these grains can only be distributed in a disk. This disk must have a large height and it must be confined to at most 10 AU. As discussed in Sect. 4.3, it is unlikely that this disk would be a gas-poor disk. Moreover, the presence of strong molecular bands in the ISO-SWS spectrum of HR 4049 (see Paper I) strongly suggests that the circumstellar environment of HR 4049 is indeed gas-rich. The gas pressure would then support the disk to keep the large scale height. The main issue to address is then: where are the small grains? Some small grains are still required for explaining the UV absorption as well as the features due to nano-diamonds and PAHs in the ISO-SWS spectrum. But there must be an efficient mechanism to concentrate the small grains in a small region through which our line-of-sight passes, while effectively removing them from the regions where most of the reprocessing of stellar radiation happens.

One way to explain this would be to assume that the small grains are a completely separate component which is not located in the disk, but above the disk or in bipolar lobes created by a more recent mass-loss event. This dichotomy between large and small grains is actually consistent with the conclusions by Joshi et al. (1987) who argued that the polarization 

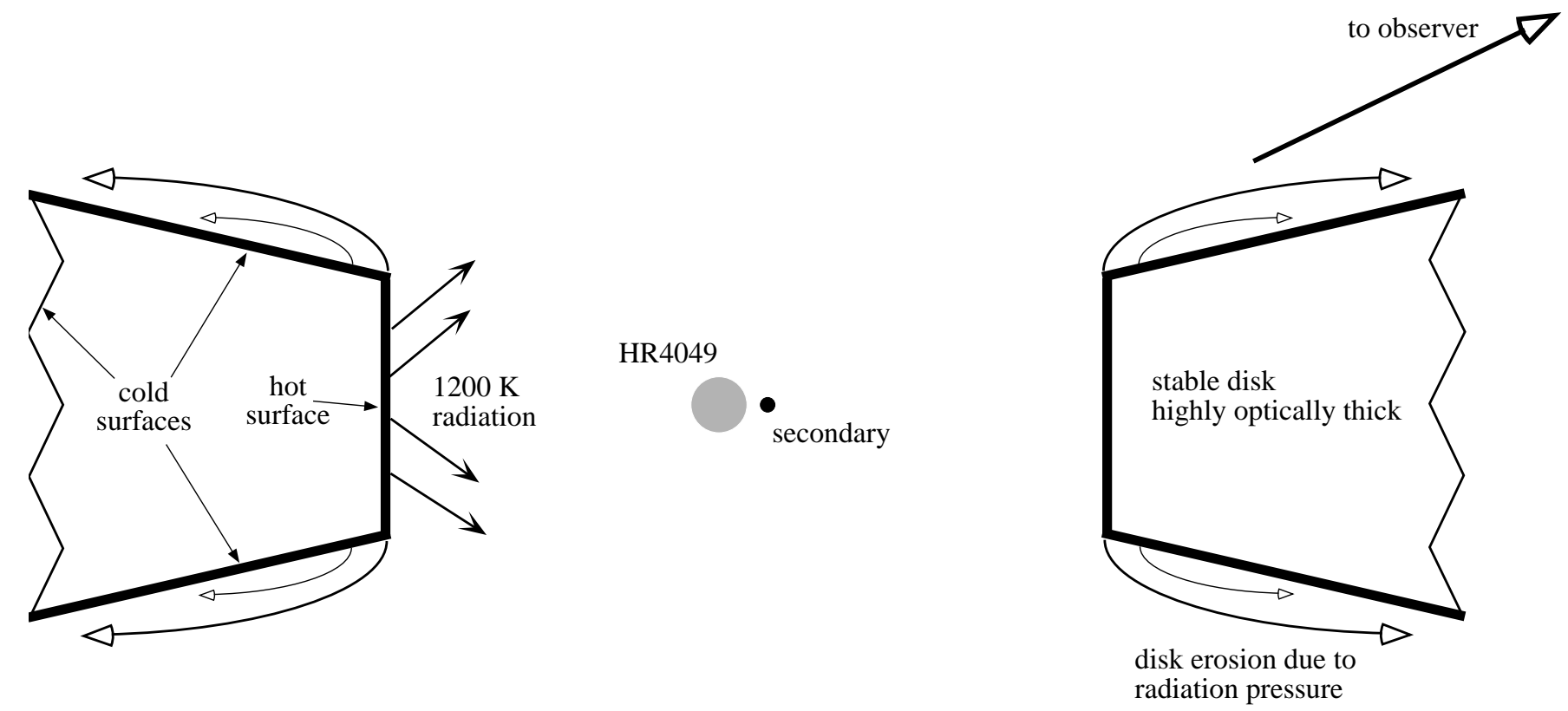

Fig. 12. Geometry sketch of the wall model for HR 4049. The double star is at the center of the fat disk. The inner surface of the disk is heated by the star and produces the blackbody radiation. The material behind the inner surface is shielded and cold, including the top, bottom and rear surfaces. The height of the disk is set by strong radiation forces which blow away the upper parts of the disk. The direction to the observer is such that we look through the edge of the disk.

they observed points to two populations of dust grains, one being very small grains, the other being large grains. However, such a geometry makes it more difficult to understand the large variations of extinction in phase with orbital variations.

As the gas which is observed through the molecular bands is typical for an O-rich environment (see Paper I), it is reasonable to assume that the dust grains which reside in this gaseous reservoir are also O-rich. If this is true, the complete lack of silicate features in the spectrum is another indication for the absence of small grains in this disk. These O-rich dust grains would then incorporate the refractory elements lacking in the photosphere of HR 4049.

If there are mainly large grains in the disk, the dust mass as derived from Eq. (10) should be a fair estimate for the total dust mass. Adopting a standard dust-to-gas ratio then yields a total disk mass of a few times $10^{-4} M_{\odot}$. This is somewhat low for the total amount of mass typically lost on the AGB. As discussed in this paper, it seems unlikely that a large fraction of the dust has escaped the system. Moreover, there is no clear-cut evidence (yet) for any kind of outflow. The rather low total disk mass would then indicate that the binary system prevented HR 4049 to have a "normal" evolution and leave the AGB prematurely, before the star could experience the most dramatic episodes of mass loss.

Apart from explaining the location of the small grains, the main problem with the large-grain model appears to be how to support the required disk height for an extended period of time. Settling (in a gas-rich disk) and collisions (in a gas-poor disk) both limit the lifetime severely.

The wall model is an appealing alternative to explain the blackbody shape of the IR continuum. In this case, a standard grain size distribution could be present in the circumbinary disk. The blackbody emission would be due to the entire surface instead of the individual grains. The visible grains near the surface all do have high temperatures of about $1200 \mathrm{~K}$, and the absence of silicate features would be due to the absence of grains in the right temperature regime, and to the small temperature gradient near the surface. The variations observed in the UV absorption - due to small grains - can be tied to the vertical density distribution. Indeed, as explained in Sect. 6.3, the differential settling times for different grain sizes would cause a layered grain size stratification in which any large grains would settle towards the midplane, while the smallest grains would still be present high in the disk, causing the extinction in a beam passing through this region. If we assume that the vertical density distribution of the small grains is not too affected by the settling mechanism, the density distribution of these small grains would follow the gas density as given in Eq. (22). As the small change in viewing angle between photometric minimum and maximum causes a large change in the amount of absorbed energy, we probably view the system with a rather high inclination, skimming just past the edge of the disk.

\subsection{The inclination of HR 4049}

We can actually get a more quantitative idea about the inclination at which we view the system by expressing it in terms of the hydrostatic scale height. From Eq. (22) it follows that

$\frac{\mathrm{d} \ln \rho}{\mathrm{d} \ln z}=-\frac{z^{2}}{h_{0}^{2}}$.

To calculate $\operatorname{dln} z$ and $\operatorname{d} \ln \rho$, we consider the following. At photometric minimum, the star is at inferior conjunction 
(i.e. closest to us, poor observers). The line of sight then crosses the disk at height $H$, thus probing material with a density $\rho_{\text {min }}$. At photometric maximum, the star is further away from us, and therefore the line of sight crosses the disk at $H+\Delta H$, this time probing material with a density $\rho_{\max }$. From simple geometric considerations it then follows that

$$
\frac{\Delta H}{H} \approx \frac{D}{R_{0}}
$$

with $D$ the distance between the star at photometric minimum and at photometric maximum and $R_{0}$ the distance between the center of mass of the binary system and the disk. At the same time, the densities are proportional to the absorption optical depth so that

$\frac{\rho_{\min }}{\rho_{\max }}=\frac{\tau_{\min }}{\tau_{\max }}$

and

$\frac{\mathrm{d} \ln \rho}{\mathrm{d} \ln z}=\frac{\mathrm{d} \rho / \rho}{\mathrm{d} z / z}=\frac{\rho_{\max }-\rho_{\min }}{\rho_{\min }} \frac{H}{\Delta H}=\left(\frac{\tau_{\max }}{\tau_{\min }}-1\right) \frac{R_{0}}{D}$.

Finally, Eq. (30) becomes

$$
\frac{z}{h_{0}}=\sqrt{\frac{R_{0}}{D}\left(1-\frac{\tau_{\max }}{\tau_{\min }}\right)} .
$$

Using the "grey" optical depths as derived from the UV absorption (see Sect. 3.1), $R_{0}=10 \mathrm{AU}$ (as in our standard model in Sect. 6) and $D \sim 1.18 \mathrm{AU}$ (van Winckel et al. 1995) we find that $z \sim 2.8 h_{0}$. With $h_{0}$ the pressure scale height (Eq. (20)) we then find that the line-of-sight crosses the disk at a height $H$ of about 5.3 AU. The inclination $i$ is then found by solving

$\tan \left(90^{\circ}-i\right)=\frac{H}{R_{0}-D / 2}$

yielding an inclination of $60^{\circ}$. This shows that the variation in the optical depth can be understood in terms of the scale height of a hydrostatic disk. It also shows that the disk must be truncated to about 2-3 pressure scale heights, consistent with what we found in Sect. 6.5. corresponding to an $H / R$ of 0.58 . However, the dynamic truncation of the disk means that the density distribution in not a perfect Gaussian. The inclination derived above is therefore an approximation.

The Wall model we presented in this paper is therefore not only capable of explaining the blackbody shape of the IR continuum, but can also explain in a consistent way the variations in the UV absorption tied to the orbital variations. In that case, the chemical composition of the dust grains and the exact geometric configuration of the different grain species observed through either the UV absorption or the features in the ISOSWS spectrum remains a matter of debate. The total mass in the circumbinary disk which is required to make the optical depths sufficiently high (see Sect. 6.2) on the other hand might well be compatible with the total mass lost during the evolution of HR 4049 on the RGB and/or the AGB taking into account that no mass has left the system.

The main difficulty with the Wall model is accurately reproducing the exact blackbody shape which we unambiguously observe. As was shown in Sect. 5.3, leakage through the top, bottom and rear surfaces of the disk still produces a significant contribution to the flux at long wavelengths, even for very high optical depths.

\subsection{The metallicity of HR 4049}

We have also derived properties concerning the disk stability. We showed that the central part of a massive disk can withstand the radiation pressure acting on the dust grains, because the radiation force will only act on the innermost grains, while the rest of the disk does not receive direct stellar radiation. It is obvious, that a much more thorough study of the dynamics of such a disk would be very interesting. Such a study requires two-fluid hydrodynamics and radiative transfer involving large optical depth. We may however speculate, that a study of the true dynamics of such a disk may provide a natural explanation for the extremely low metallicity of HR 4049. Envision the disk filled with small grains. The radiation pressure of the star will push the grains into the inner rim of the disk, leading to very high dust densities there. The dust grains will collide with the gas and provide an outward force to the entire medium. But since the outer disk does not receive direct stellar light, it is not moving radially. Therefore, either a large radial pressure gradient or substantial sub-Keplerian rotation must balance the radiation force. However, we have shown in Sect. 6.5 that the grains will slowly drift outwards through the gas, reducing the dust content in the innermost layers. Once a layer of gas has become dust free, the radiation force acting on this layer will become very small. The layer falls inwards, driven either by the gas pressure gradient, or by its sub-Keplerian rotation. The falling gas will be free of dust and therefore have very low metal abundances. If this gas can eventually be reaccreted by the primary of HR 4049, it will form a metal-poor photospheric layer on the star. Since the disk may contain a significant fraction of a solar mass, a substantial amount of metal free gas is available for this mechanism.

\subsection{Fat circumstellar disks in post-AGB binary stars}

Geometrically thick circumstellar disks are not uncommon in post-AGB stars. Similar to HR 4049 are objects with a detected binary with a rather small orbital period implying strong binary interaction during evolution. A famous example is the Red Rectangle (HD 44179), where the disk is even resolved (Roddier et al. 1995). The Red Rectangle also shows variability in phase with the orbital motion of the binary, but the variability is entirely grey. Waelkens et al. (1996) explain the variability in this source with a variable angle under which the stellar light is scattered towards the observer. This model also requires the presence of a fat disk around the system, similar to our proposed wall model. Another example is HD 213985, which shows the same photometric behavior as HR 4049 (Waelkens et al. 1995). The link to the central binary characteristics is less clear for objects where the disc is resolved like the Egg Nebula (Sahai et al. 1998). The nature of the central binary in this object is not yet known. 
However, none of the above sources shows the perfect blackbody shape of the excess emission.

\section{Conclusions}

The Spectral Energy Distribution of HR 4049 shows three significant keys which can be used to determine the basic properties of its circumstellar dust: a variable, non-grey circumstellar extinction, an infrared excess which amounts to $1 / 3$ of the stellar flux and the perfect blackbody shape of the excess between $1 \mu \mathrm{m}$ and $850 \mu \mathrm{m}$. We conclude that the bulk of the circumstellar matter must be in a disk with large scaleheight $H / R \sim 1 / 3$. We have considered large grains in a marginally optically thick disk as a possible explanation and find that such a structure is subject to very tight constraints on the radial extent and optical depth of the disk, and that the large scale height is difficult to support for an extended time. We propose, as an alternative solution, the wall model which is a fat disk with a radial optical depth in excess of $10^{4}$. The wall model fulfills many of the requirements, with the only significant limitation that the optical depth must be very high to suppress excess flux at $850 \mu \mathrm{m}$. The wall can explain the observed variable extinction in a very consistent way, while the big-grain model requires a separate component for this observation. In the context of binary evolution, the formation of a massive circum-binary disk can be qualitatively understood as the result of tidal interaction during the AGB phase of the primary. The wall model is therefore an attractive candidate to describe the CS environment of HR 4049.

In the near future, the MIDI interferometer at the VLT should be able to resolve the disk around HR 4049. Wavelength dependent interferometric measurements will give information about the location of PAH emission and continuum, and about the location of the inner disk rim. HR 4049 has fascinated astronomers now for about 20 years - there is more to come.

Acknowledgements. We would like to thank Rens Waters, Xander Tielens and Jorge Jimenez Vicente for stimulating and insightful discussions. We would like to thank Remo Tilanus for the SCUBA observations and the data reduction. $\mathrm{CD}$ and JC acknowledge the financial support from NWO Pionier grant 6000-78-333. CPD and CD acknowledge support from the European Commission under TMR grant ERBFMRX-CT98-0195 ("Accretion onto black holes, compact objects and protostars").

\section{References}

Backman, D. E., \& Paresce, F. 1993, in Protostars and Planets III, ed. E. H. Levy, \& J. I. Lunine (Tuscon, University of Arizona Press), 1253
Bakker, E., Lambert, D., van Winckel, H., et al. 1998, A\&A, 336, 263 Beintema, D., van den Ancker, M., Molster, F., et al. 1996, A\&A, 315, L369

Buss, R. H., Snow, T. P., \& Lamers, H. J. G. L. M. 1989, ApJ, 347, 977

Chen, C. H., \& Jura, M. 2001, ApJ, 560, L171

Chiang, E. I., \& Goldreich, P. 1997, ApJ, 490, 368

Cuzzi, J. N., Durisen, R. H., Burns, J. A., \& Hamill, P. 1979, Icarus, 38,54

de Graauw, T., Haser, L. N., Beintema, D. A., et al. 1996, A\&A, 315, L49

Dominik, C., Gail, H. P., \& Sedlmayr, E. 1989, A\&A, 223, 227

Draine, B. T., \& Lee, H. M. 1984, ApJ, 285, 89

Dullemond, C. P., Dominik, C., \& Natta, A. 2001, ApJ, 560, 957

Geballe, T., Noll, K., Whittet, D., \& Waters, L. 1989, ApJ, 340, L29

Guillois, O., Ledoux, G., \& Reynaud, C. 1999, ApJ, 521, L133

Icke, V. 1988, 202, 177

Jeffreys, H. 1947, MNRAS, 107, 263

Johnson, J. J., Anderson, C. M., Bjorkman, K. S., et al. 1999, MNRAS, 306,531

Joshi, U. C., Deshpande, M. R., Sen, A. K., \& Kulshrestha, A. 1987, A\&A, 181, 31

Kenyon, S. J., \& Hartmann, L. 1987, ApJ, 323, 714

Kessler, M. F., Steinz, J. A., Anderegg, M. E., et al. 1996, A\&A, 315, L27

Lamers, H., Waters, L., Garmany, C., Perez, M., \& Waelkens, C. 1986, A\&A, 154, L20

Mellema, G. 1997, A\&A, 321, L29

Miyake, K., \& Nakagawa, Y. 1995, ApJ, 441, 361

Monier, R., \& Parthasarathy, M. 1999, A\&A, 341, 117

Press, W. H., Teukolsky, S. A., Vetterling, W. T., \& Flannery, B. P. 1992, Numerical recipes in FORTRAN, The art of scientific computing (Cambridge: University Press), 2nd ed.

Pringle, J. E. 1981, ARA\&A, 19, 137

Roddier, F., Roddier, C., Graves, J. E., \& Northcott, M. J. 1995, ApJ, 443,249

Rybicki, G. B., \& Lightman, A. P. 1979, Radiative Processes in Astrophysics (Johhn Wiley \& Sons)

Sahai, R., Trauger, J. T., Watson, A. M., et al. 1998, ApJ, 493, 301

Savage, B. D., \& Mathis, J. S. 1979, ARA\&A, 17, 73

Tielens, A. G. G. M., McKee, C. F., Seab, C. G., \& Hollenbach, D. J. 1994, ApJ, 431, 321

van Winckel, H. 2001, Ap\&SS, 275, 159

van Winckel, H., Waelkens, C., \& Waters, L. 1995, A\&A, 293, L25

Waelkens, C., Lamers, H., Waters, L., et al. 1991, A\&A, 242, 433

Waelkens, C., Van Winckel, H., Waters, L. B. F. M., \& Bakker, E. J. 1996, A\&A, 314, L17

Waelkens, C., Waters, L. B. F. M., Van Winckel, H., \& Daems, K. 1995, Ap\&SS, 224, 357

Waters, L. B. F. M., Lamers, H. J. G. L. M., Snow, T. P., et al. 1989, A\&A, 211, 208

Waters, L. B. F. M., Trams, N. R., \& Waelkens, C. 1992, A\&A, 262, L37

Zuckerman, B., \& Becklin, E. E. 1993, ApJ, 406, L25 